\title{
A Statistical Dictionary Approach to Automated Orientation Determination from Precession Electron Diffraction Patterns
}

\author{
A. Wang ${ }^{1}$, A.C. Leff $^{2}$, M.L. Taheri ${ }^{2}$ and M. De Graef ${ }^{1}$ \\ 1. Dept. of Materials Science and Engineering, Carnegie Mellon University, Pittsburgh PA 15213, USA \\ 2. Dept. of Materials Science and Engineering, Drexel University, Philadelphia PA 19104, USA
}

The precession electron diffraction (PED) technique has been widely used for structure determination since Vincent and Midgley proposed it in 1994 [1]. By acquiring electron diffraction patterns using electron beam precession, the usually strong dynamical scattering effects can be averaged out. Moreover, PED patterns can be indexed with a higher angular resolution than conventional diffraction patterns since more reflections can be collected. In order to determine the crystal orientation from a PED pattern, one would need to measure distances and angles between diffraction spots, a process that rapidly becomes tedious for multigrain samples. A faster approach would be to use a physics-based model to produce simulated diffraction patterns which are pre-calculated according to the microscope settings and the crystal parameters; this is known as a dictionary-based approach. The crystal orientation for a given PED pattern is then obtained by finding the best match against the dictionary. Rauch et al. [2] have developed an automated orientation and phase mapping method in TEM by using PED, which has been implemented in NanoMEGAS' commercial software ASTAR. This method makes use of template-matching algorithms, where the templates are pre-calculated based on the crystal symmetry using kinematical scattering within the fundamental zone in Euler angle space. The locations and intensities of the diffraction spots in an experimental PED pattern are then cross-correlated with the template patterns to find the best match. However, the extensive use of image filtering in ASTAR can make the indexing process somewhat less reproducible because the filtering parameters used prior to cross-correlation may yield variable indexing results. Additionally, the method described in [2] does not always reliably identify orientations from patterns taken on or near grain boundaries because diffraction spots from both orientations are present in the patterns.

In [3], an alternative and simple indexing framework based on normalized inner products between the experimental and dictionary patterns was proposed. This approach can determine the crystal orientation but can also differentiate the grain interior from the grain boundary without extensive image processing. Moreover, this approach has the advantage of operating on the raw data, without prior application of image processing tools. The first step is the computation of normalized inner products for each experimental PED pattern with all the dictionary patterns. The set of Euler angles of an experimental pattern is then obtained from the dictionary pattern that results in the largest inner product. The second step is to use a decision tree classifier to cluster the diffraction patterns using the statistical distribution of the normalized inner products and then to segment the grains and identify anomalous regions. This technique was successfully demonstrated with EBSD indexing for a Ni-base alloy. In the present study, we apply the method described in [3] to index PED patterns in a $\mathrm{Cu}$ sample containing a twin boundary and compare the results with those obtained using the ASTAR approach.

Fig. 1.(a) shows a virtual bright field image constructed from PED patterns acquired across an area of $60 \times 80$ samples, using a $10.4 \mathrm{~nm}$ step size. The data was acquired using a JEOL $2100 \mathrm{LaB}_{6}$ operated at $200 \mathrm{keV}$, using a $10 \mathrm{~nm}$ spot size and a precession angle of $0.60^{\circ}$. An example PED pattern acquired from 
the upper grain and its best match from the dictionary are presented in Fig. 1.(b) and (c), respectively. As shown in Fig. 1.(d), the image pixels are clustered well to differentiate the two grains and the twin boundary is clearly visible. Also, the sample edge is clearly defined. The Euler angle results obtained from the dictionary approach are shown in Fig. 1.(e). Based on the two cluster angles, the misorientation between the two grains is a $62.55^{\circ}$ rotation about the $[0.62,0.56,0.55]$ axis $\left(3.4^{\circ}\right.$ from $\left.[111]\right)$, veryfying the boundary to be a twin boundary. The $y$-axis orientation map obtained from ASTAR is shown in Fig. 1.(f); the edge of the sample is not visible, presumably due to residual intensity from the screen phosphor for beam positions outside of the sample. Our dictionary approach shows promising results for the indexing of orientations from PED patterns. Further investigations for multigrain thin films are currently ongoing.

\section{References:}

[1] R. Vincent and P.A. Midgley, Ultramicroscopy 53 (1994), p.271.

[2] E.F. Rauch and M. Veron, Materwiss. Werksttech. 36 (2005), p.552

[3] Y.H. Chen, S.U. Park, D. Wei et. al., Microscopy and Microanalysis, under review.

[4] A. Wang and M. De Graef are grateful for AFOSR support under MURI contract \#FA9550-12-1-0458. A.C. Leff and M.L. Taheri are grateful for support from: US DOE Basic Energy Sciences Early Career program (DE-SC0008274); National Science Foundation Faculty Early Career Program (\#1150807); and DOE Nuclear Energy University Program (NE0000315).
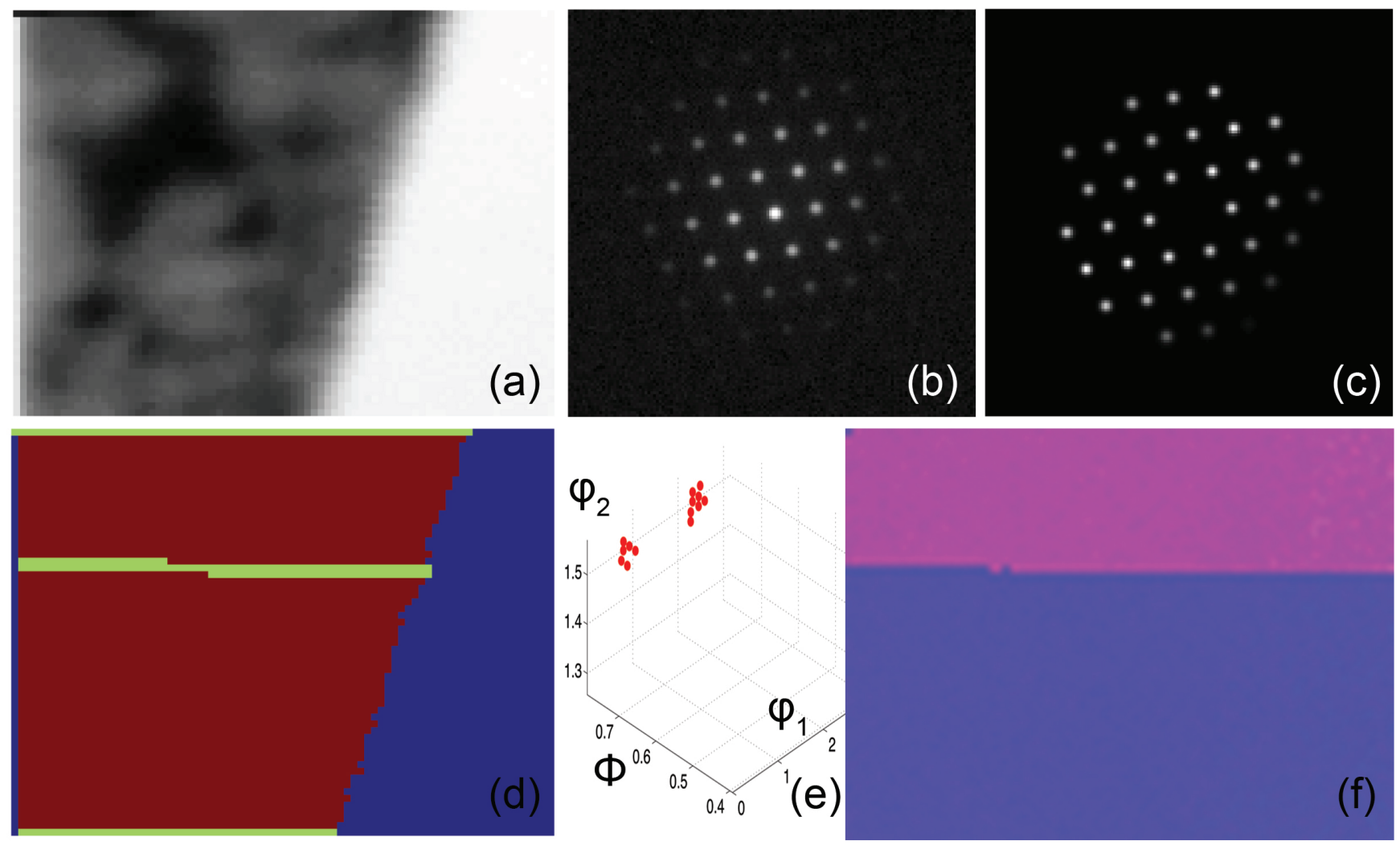

Figure 1. (a) Virtual bright field image of a $\mathrm{Cu}$ sample with a twin boundary, image area $60 \times 80$ pixels, pixel size $10.4 \mathrm{~nm}$; (b) Experimental PED pattern from the upper grain; (c) Best matching pattern from the dictionary; (d) Color map resulting from the cluster classifier (red pixels are grain interior, light green twin boundary, blue vacuum area); (e) Euler angle distribution, showing two clusters; (f) ASTAR color map. 